\title{
ADNCR modulates neural stem cell differentiation and proliferation through the regulation of TCF3 expression
}

\author{
Ling Long ${ }^{1 *}$, Chao Zeng ${ }^{2 *}$, Honglei Chen ${ }^{3}$, Taicheng $\mathrm{Zhou}^{4}$, Lili Wu ${ }^{5}$, Xiaodong Cai ${ }^{6}$ \\ ${ }^{1}$ Department of Neurology, the Third Affiliated Hospital of Sun Yat-sen University, Guangzhou, China; ${ }^{2}$ Department of Pathology, the Eighth \\ Affiliated Hospital of Sun Yat-sen University, Shenzhen, China; ${ }^{3}$ Department of Gastrointestinal Endoscopy, Guangdong Provincial Key \\ Laboratory of Colorectal and Pelvic Floor Diseases, the Sixth Affiliated Hospital of Sun Yat-sen University, Guangzhou, China; ${ }^{4}$ Department \\ of Gastroenterological Surgery and Hernia Center, the Sixth Affiliated Hospital of Sun Yat-sen University, Guangzhou, China; ${ }^{5}$ Department of \\ Ultrasound, the Third Affiliated Hospital of Sun Yat-sen University, Guangzhou, China; ${ }^{6}$ Department of Neurology, the Sixth Affiliated Hospital of \\ Sun Yat-sen University, Guangzhou, China \\ Contributions: (I) Conception and design: L Long, X Cai; (II) Administrative support: X Cai; (III) Provision of study materials: C Zeng, H Chen; (IV) \\ Data analysis and interpretation: T Zhou, L Wu, X Cai; (V) Manuscript writing: All authors; (VI) Final approval of manuscript: All authors. \\ \#These authors contributed equally to this work. \\ Correspondence to: Xiaodong Cai, MD, PhD. Department of Neurology, the Sixth Affiliated Hospital of Sun Yat-sen University, No. 26, Yuancun II \\ Heng Road, Guangzhou, China. Email: caixd3@mail.sysu.edu.cn.
}

Background: Neural stem cells (NSCs) are undifferentiated precursor cells that have the ability to selfrenew and proliferate and have the capacity to become either glia (oligodendrocytes and astrocytes) or neurons. NSCs can act as beneficial adjuncts for many neurological disorders, such as cerebral infarction, spinal cord injuries, Alzheimer's disease, and Parkinson's disease. Long noncoding RNAs (lncRNAs) play essential roles during cell differentiation, proliferation, and metabolism. This study aimed to explore the role played by adipocyte differentiation-associated long noncoding RNA (ADNCR) in the self-renewal and multipotency of NSCs.

Methods: In this study, we identified NSCs and verified that these cells were able to regenerate and differentiate into both astrocytes and neurons. Then we studied the relation between expression of ADNCR and transcription factor 3 (TCF3) and proliferation of NSCs.

Results: ADNCR and TCF3 expression have been shown to decrease during the differentiation of NSCs into both neurons and astrocyte induction cells. However, the expression of the microRNA miR204-5p increased over time during the differentiation of NSCs into both neurons and astrocyte induction cells. ADNCR acts as a competing endogenous RNA (ceRNA) for miR-204-5p, and the overexpression of ADNCR suppressed miR-204-5p expression and enhanced TCF3 expression in NSCs, which resulted in enhanced proliferation and suppressed neural differentiation.

Conclusions: These data suggested that the use of ADNCR may represent a new strategy for expanding the interventions used to treat neurological disorders.

Keywords: Adipocyte differentiation-associated long noncoding RNA (ADNCR); long noncoding RNAs (lncRNAs); transcription factor 3 (TCF3); miR-204-5p

Submitted Jan 28, 2020. Accepted for publication Jul 09, 2020.

doi: $10.21037 /$ atm-20-1068

View this article at: http://dx.doi.org/10.21037/atm-20-1068 


\section{Introduction}

Neural stem cells (NSCs) are undifferentiated precursor cells that have the ability to self-renew and proliferate and have the capacity to become either glia (oligodendrocytes and astrocytes) or neurons (1-3). NSCs have been widely investigated for potential clinical uses (4-6). Several studies have suggested that NSCs can act as beneficial adjuncts and could potentially be used to treat for many neurological disorders, such as cerebral infarction, spinal cord injuries, Alzheimer's disease, Parkinson's disease, epilepsy, and neurasthenia (4,7-10). Although the functional characteristics of NSCs have been widely investigated, the molecular mechanisms underlying NSC migration, differentiation and self-renewal remain unknown (11-13). Thus, studying the molecular mechanisms that modulate migration, differentiation and self-renewal is crucial to understanding the determinants of NSC cellular fates.

Long noncoding RNAs (lncRNAs) are RNAs that are longer than two hundred nucleotides (nts) in length and belong to one class of noncoding RNAs (ncRNAs) (14-17). Recent studies have revealed that several lncRNAs play essential roles in a large range of functions, including cell fate, differentiation, proliferation, invasion, and metabolism (18-21). The expression of many lncRNAs was found to be dysregulated during human diseases, such as tumor, intervertebral disc degeneration, spinal cord injuries, and Parkinson's disease (22-25). Recently, growing evidence has suggested that lncRNAs also play roles in the development, self-renewal, and differentiation of NSCs (26-28). A new lncRNA, adipocyte differentiation-associated long noncoding RNA (ADNCR), was recently identified and found to suppress the differentiation of adipocytes (29). However, the role played by ADNCR in the self-renewal and multipotency of NSCs remains unknown.

In our study, we first identified NSCs and found that these cells could regenerate and differentiate into both astrocytes and neurons. ADNCR expression was found to decrease during the differentiation of NSCs into both neurons and astrocyte induction cells. The ectopic expression of ADNCR suppressed the proliferation of NSCs and the differentiation of NSCs into neurons.

\section{Methods}

\section{Cell culture and transfection}

Primary NSCs were cultured using a modified method based on previously published protocols $(26,30)$. Primary
NSCs were isolated from 13.5-day rat embryos and maintained in DMEM medium, supplemented with N2, epidermal growth factor (EGF) and basic fibroblast growth factor (bFGF, R\&D). pcDNA-control, pcDNA-ADNCR, miR-NC, miR-204-5p, siRNA-control, and siRNAtranscription factor 3 (TCF3) were acquired from Ambion (Thermo). These vectors were transfected into primary NSCs using Lipofectamine 3000 (Invitrogen, USA), according to the manufacturer's instructions.

\section{Immunocytochemistry}

Cells were first fixed with $4 \%$ paraformaldehyde and permeabilized with Triton-X100 (0.2\%). After blocking in $10 \%$ serum, the cells were treated with mouse anti$\beta$-tubulin III monoclonal antibody (clone SDL.3D10, RRID:AB_2210370; Catalog No. T8660; Sigma-Aldrich), at $4{ }^{\circ} \mathrm{C}$ for 12 hours, and followed by incubation with secondary antibodies.

\section{Cell proliferation}

Cell growth was determined using Cell Counting kit 8 (CCK8) analysis (Dojindo, Japan), according to the manufacturer's instructions. The growth rate was analyzed at different time points after transfection. The absorbance was detected at $450 \mathrm{~nm}$ by a microplate reader.

\section{qRT-PCR}

Total RNA was extracted from the cells using a TRIzol kit (Invitrogen, CA, USA). The expression levels of miRNA, IncRNA and mRNA were determined by quantitative real-time reverse transcription polymerase chain reaction (qRT-PCR) analysis using SYBR Green (QIAGEN, Germany) on a 7500 PCR detection system (Applied Biosystems, Thermo). The primers used were as follows: $\beta$-tubulin III, 5'-AGCAAGGTGCGTGAGGAGTA-3' and 5'-TCTAGTGTCTCATGGCTCTGGTTTT-3'; Nestin, 5'-GATCTAAACAGGAAGGAAATCCAGG-3' and 5'-TCTAGTGTCTCATGGCTCTGGTTTT-3'; GAPDH, 5'-CTCCTCCTGTTCGACAGTCAGC-3' and $5^{\prime}$-CCCAATACGACCAAATCCGTT-3'. The data are shown as $2^{-\triangle \Delta C T}$ means.

\section{Western blot}

Cells were lysed with cell lysis buffer (Pierce, Thermo 
A

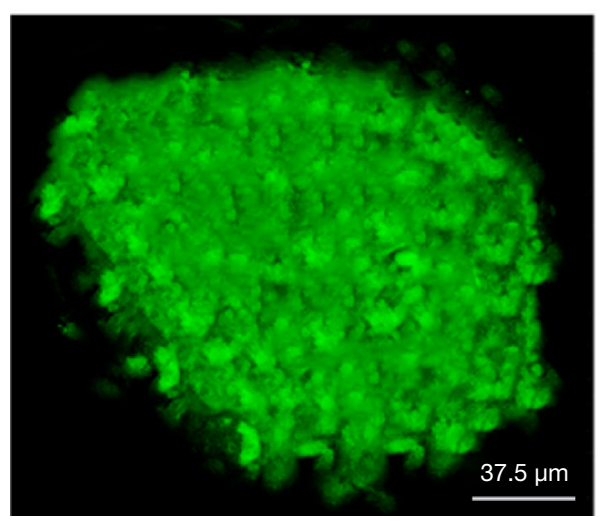

B

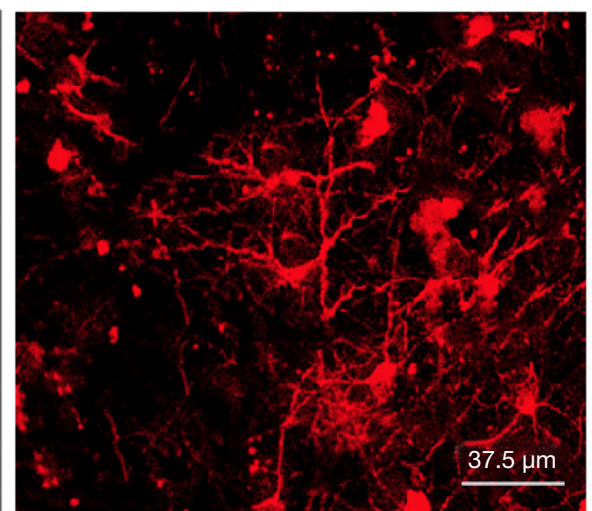

C

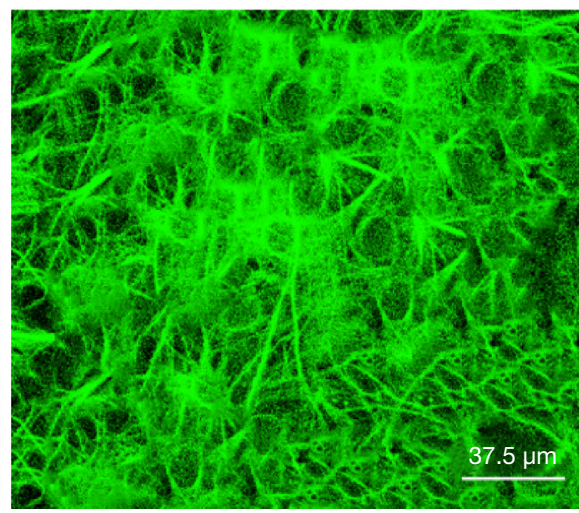

Figure 1 NSCs have regenerative abilities and can differentiate into astrocytes and neurons. (A) Cells were immunostained with the NSCmarker nestin; (B) differentiated cells, immunostained with the neuron-specific marker $\beta$-tubulin III; (C) differentiated cells, immunostained with the astrocyte-specific marker GFAP. NSC, neural stem cell; GFAP, glial fibrillary acidic protein.

Fisher Scientific) to obtain total protein. Protein was resolved on a $10 \%$ SDS-PAGE gel and then transferred into a polyvinylidene difluoride (PVDF) membrane. The membrane was blocked with milk for 2 hours and then blotted with mouse anti- $\beta$-tubulin III monoclonal antibody (clone SDL.3D10, RRID: AB_2210370; Catalog No. T8660; Sigma-Aldrich), mouse anti-TCF3 monoclonal antibody (clone 5G2, RRID: AB_2255610; Catalog No. SAB1404452; Sigma-Aldrich) and Mouse anti-GAPDH monoclonal antibody (clone CL3266, RRID: AB_10597731; Catalog No. AMAB91153; Sigma-Aldrich). The blot was incubated with secondary antibodies, and an ECL kit (Millipore, USA) was used to visualize the proteins. GAPDH was used as a loading control.

\section{Luciferase reporter assay}

The mutant (Mut) or wild-type (WT) 3'UTR of TCF3 was cloned into the pGL3-luciferase reporter vector (Promega, USA). NSCs were co-transfected with miR-204-5p mimic or miR-NC, and Mut or WT TCF3 3'UTR, and Renilla luciferase (Promega, USA), using Lipofectamine 3000 (Invitrogen, USA). Luciferase activity was determined with the Dual-Luciferase kit, according to the manufacturer's instructions.

\section{Statistical analysis}

The results are presented as the mean \pm SD (standard deviation), and statistical analyses were performed using SPSS. Student's $t$-test was used to measure significant differences between two groups. A P value of $<0.05$ was defined as statistically significant.

\section{Results}

\section{NSCs bave regenerative abilities and can differentiate into astrocytes and neurons}

Isolated NSCs have regenerative abilities and can be shaped into neurospheres, which were positive for nestin (Figure 1A), which is a specific marker for NSCs. After the removal of bFGF, FBS, and EGF, the NSCs differentiated into astrocytes and neurons, which were identified by immunostaining using the neuron-specific marker $\beta$-tubulin III (Figure $1 B$ ) and the astrocyte-specific marker glial fibrillary acidic protein (GFAP) (Figure 1C).

\section{Decreased ADNCR and TCF3 and increased miR-204-5p expression levels during NSC differentiation}

To study the functional role of ADNCR during the differentiation of NSCs, we measured ADNCR expression levels during NSC differentiation using qRT-PCR. The data showed that ADNCR expression decreased during the differentiation of NSCs into both neurons (Figure $2 A$ ) and astrocytes (Figure 2B). In addition, the expression level of miR-204-5p increased over time during the differentiation 
of NSCs into both neurons (Figure 2C) and astrocytes (Figure 2D). Moreover, TCF3 was downregulated during the differentiation of NSCs into both neurons (Figure 2E) and astrocytes (Figure 2F).

\section{ADNCR acts as a competing endogenous RNA (ceRNA) for miR-204-5p and regulates TCF3 expression}

ADNCR expression was upregulated in NSCs transfected with the pcDNA-ADNCR vector (Figure $3 A$ ). The miR204-5p expression levels increased in the NSCs after transfection with the miR-204-5p mimic (Figure 3B). We used bioinformatics analysis to show that TCF3 is a potential target for miR-204-5p (Figure 3C). The dualluciferase reporter assay showed that the ectopic expression of miR-204-5p decreased the luciferase activity of WT TCF3 3'-UTR but not Mut TCF3 3'-UTR (Figure 3D). The overexpression of ADNCR inhibited miR-204-5p expression, according to the qRT-PCR analysis (Figure $3 E$ ). The ectopic expression of ADNCR enhanced TCF3 expression, as assessed by western blot (Figure 3F).

\section{The ectopic expression of ADNCR promoted cell proliferation and suppressed the neuronal differentiation of NSCs}

As shown in Figure 4A, the elevated expression of ADNCR enhanced NSC growth, as assessed by the CCK-8 assay. In addition, ectopic ADNCR expression increased nestin expression in NSCs (Figure 4B). Furthermore, our data showed that $\beta$-Tubulin III expression was inhibited in neural induction cells treated with pcDNA-ADNCR (Figure 4C,D). The immunocytochemical staining of $\beta$-Tubulin III also indicated that the overexpression of ADNCR suppressed NSC neuronal differentiation (Figure 4E).

\section{The inhibition of TCF3 expression abolishes the effects of ADNCR overexpression on NSC differentiation and proliferation}

To further confirm the contributions of TCF3 to the functions of ADNCR during NSC differentiation and proliferation, we suppressed TCF3 expression in ADNCRoverexpressing NSCs. As shown in Figure 5A, TCF3 expression was downregulated in NSCs after treatment with siRNA-TCF3. We also found that the protein expression of TCF3 was decreased in NSCs after treatment with
siRNA-TCF3 (Figure 5B). By using CCK-8 analysis, the results indicated that the knockdown of TCF3 expression suppressed cell growth in ADNCR-overexpressing NSCs (Figure 5C). The inhibition of TCF3 expression also decreased nestin expression in ADNCR-overexpressing NSCs (Figure 5D). Furthermore, the knockdown of TCF3 expression promoted $\beta$-Tubulin III expression in ADNCR-overexpressing NSCs (Figure 5E). In addition, the immunocytochemical staining of $\beta$-Tubulin III also showed that the suppression of TCF3 enhanced NSC differentiation into neurons (Figure 5F).

\section{Discussion}

In our study, we first identified NSCs and determined that these cells have regenerative abilities and can differentiate into astrocytes and neurons. ADNCR and TCF3 expression levels decreased during the differentiation of NSC into both neural and astrocyte induction cells. However, the expression of miR-204-5p increased over time during the differentiation of NSCs into both neural and astrocyte induction cells. The ectopic expression of ADNCR induced cell proliferation and suppressed the neuronal differentiation of NSCs. ADNCR acts as a ceRNA for miR-204-5p, and the overexpression ADNCR suppressed miR-204-5p expression and enhanced TCF3 expression in NSCs. The ectopic expression of ADNCR induced NSC proliferation and suppressed the neuronal differentiation of NSCs, partly by regulating miR-204-5p/TCF3 expression.

NSCs share two requisite properties with all stem cells, self-renewal, and multipotency, and they can differentiate into both astrocytes and neurons $(3,31,32)$. The differentiation and maintenance of NSCs are tightly regulated by molecular networks $(33,34)$. Recently, the functions of lncRNAs during the control of NSCs selfrenewal and multipotency has been investigated. Zhang et al. (35) showed that the lncRNAs Rik-203 and Rik-201 suppressed neural differentiation via the regulation of miR$467 a-3 p$ and miR-96, respectively. Li and colleagues found that the lncRNA lnc158 increased the differentiation of neural precursor cells into oligodendrocytes by modulating nuclear factor-IB (36). Winzi et al. demonstrated that the lncRNA lncR492 suppressed embryonic stem cell differentiation into neurons (37). In addition, Li et al. reported that the novel lncRNA ADNCR suppressed the differentiation of adipocytes (29). However, the role played by ADNCR in the self-renewal and multipotency of NSCs 
A

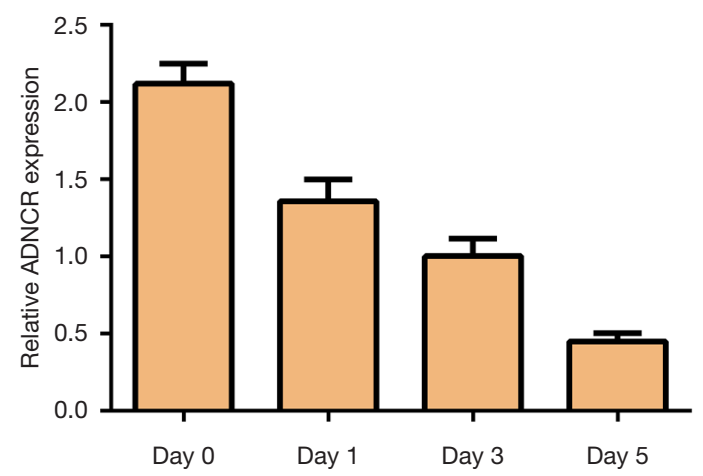

C

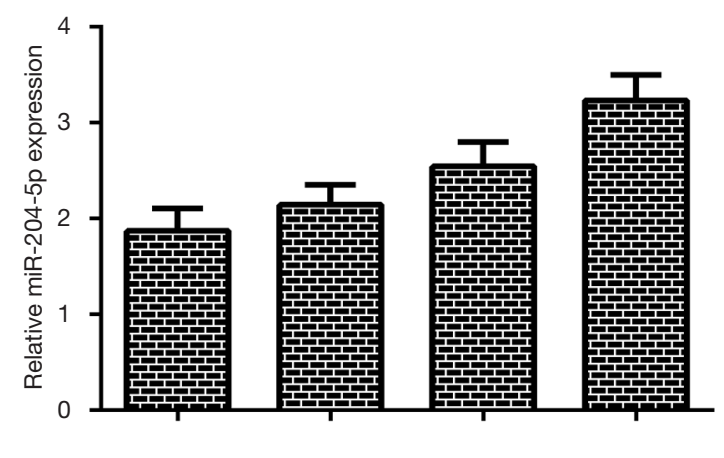

$\begin{array}{llll}\text { Day } 0 & \text { Day } 1 & \text { Day } 3 & \text { Day } 5\end{array}$

E

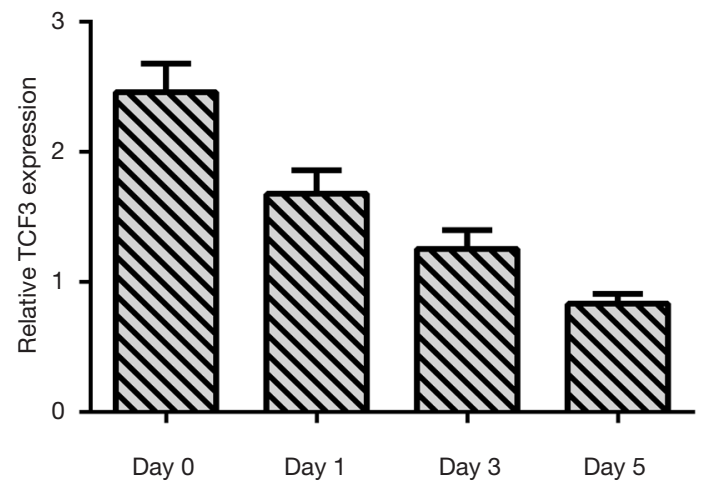

B

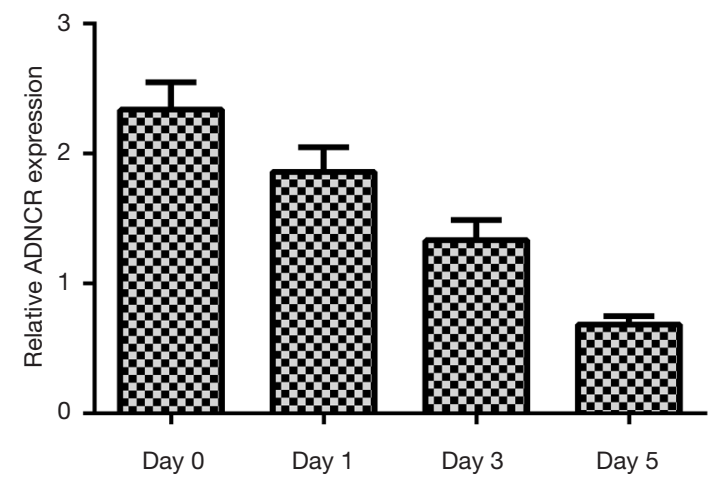

D

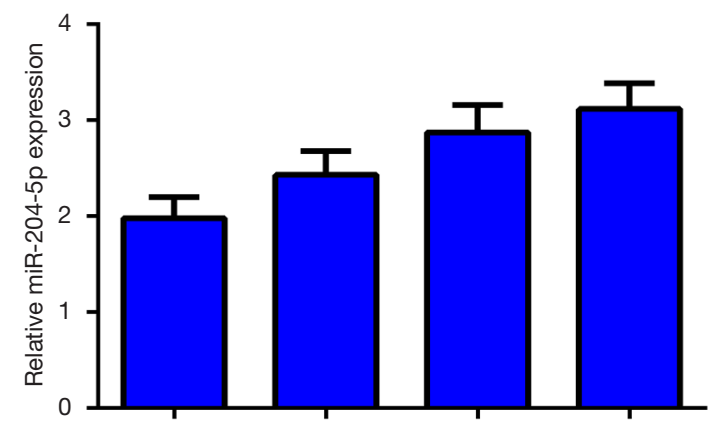

Day 0 Day $1 \quad$ Day 3 Day 5

$\mathrm{F}$

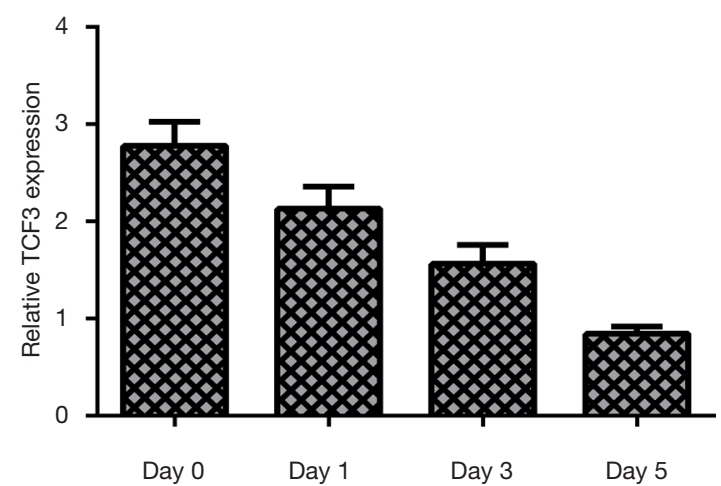

Figure 2 Decreased ADNCR and TCF3 and increased miR-204-5p expression levels during the differentiation of NSCs. (A) ADNCR expression was decreased during the differentiation of NSCs into neuron induction cells; (B) the expression of ADNCR was measured by qRT-PCR assay; (C) the expression levels of miR-204-5p increased over time during the differentiation of NSCs into neuron induction cells; (D) the expression of miR-204-5p was measured by qRT-PCR assay; (E) the expression level of TCF3 was decreased during the differentiation of NSCs into neuron induction cells; (F) the expression of TCF3 was determined by qRT-PCR assay. ADNCR, adipocyte differentiation-associated long noncoding RNA; NSC, neural stem cell; TCF3, transcription factor 3. 
A

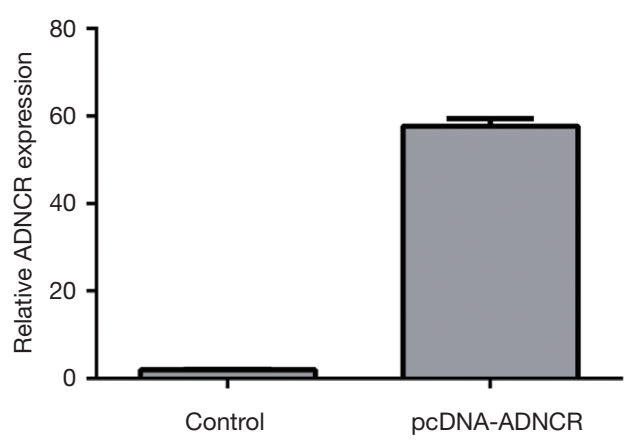

C

\section{5 '-GGAGUUUGAUCUCUUAAGGGAAC 3' UTR WT 3'-UCCGUAUCCUACUGUUUCCCUU miR-204-5p 5'-GGAGUUUGAUCUCUUUUCCCUAC 3' UTR Mut}

$\mathrm{E}$

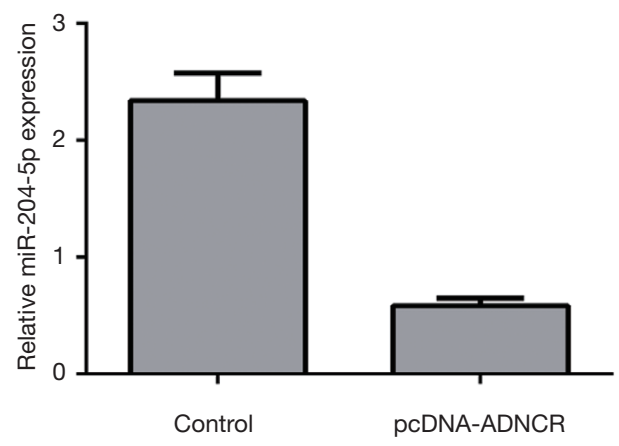

B

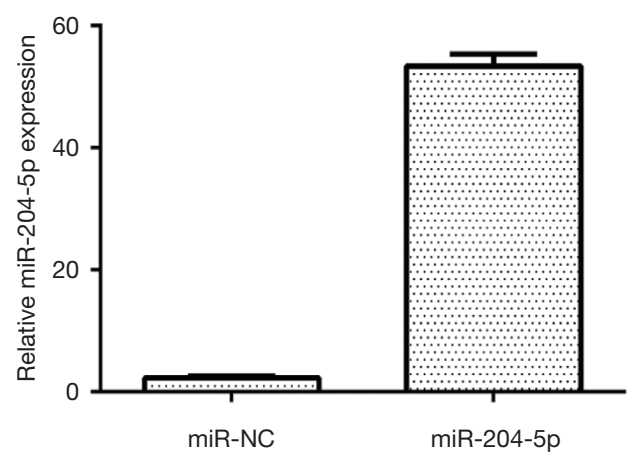

D

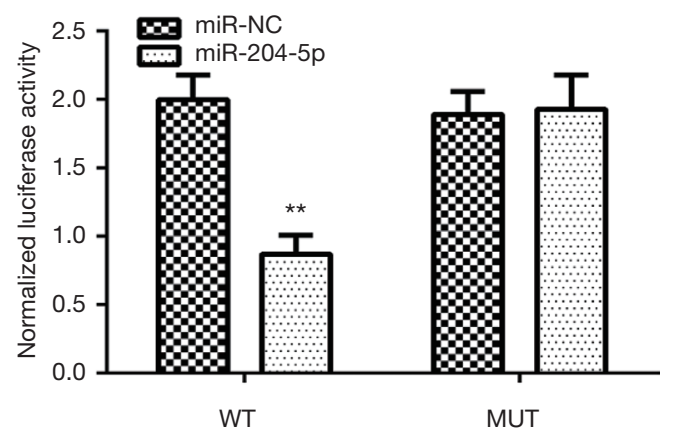

$\mathrm{F}$

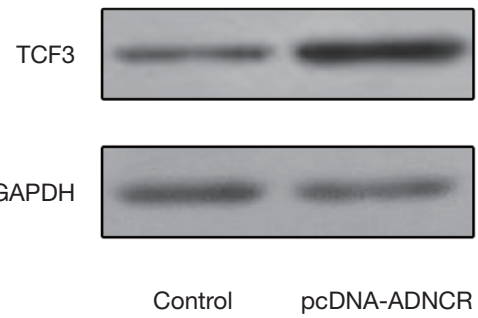

Figure $3 \mathrm{ADNCR}$ acts as a ceRNA for miR-204-5p and regulates TCF3 expression. (A) The expression of ADNCR was detected by qRTPCR assay; (B) the expression of miR-204-5p was measured by qRT-PCR assay; (C) TCF3 is a potential target of miR-204-5p, according to the results of the dual-luciferase reporter assay; (D) the ectopic expression of miR-204-5p decreased luciferase activity of WT (wild-type) TCF3 3'-UTR but not Mut (mutated) TCF3 3'-UTR; (E) the overexpression of ADNCR inhibited miR-204-5p expression, as assessed by qRT-PCR; (F) the ectopic expression of ADNCR enhanced TCF3 expression, as assessed by western blot. **, $\mathrm{P}<0.01$. ADNCR, adipocyte differentiation-associated long noncoding RNA; TCF3, transcription factor 3. 
A

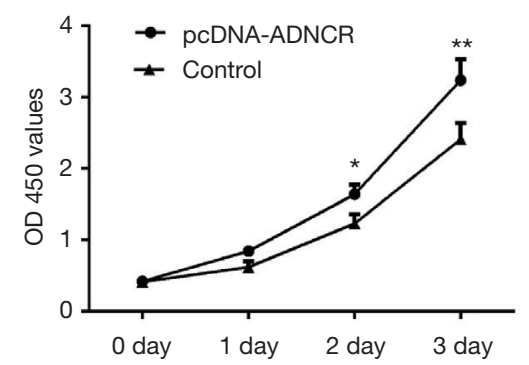

D

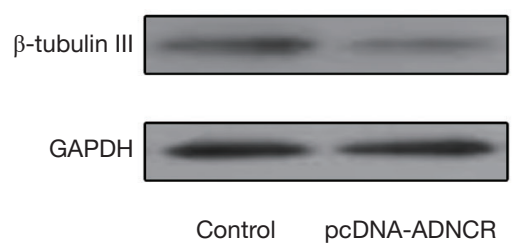

B

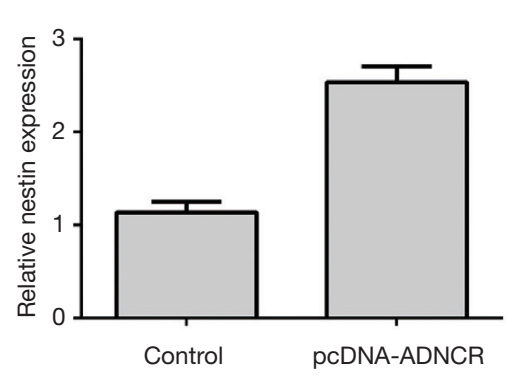

C

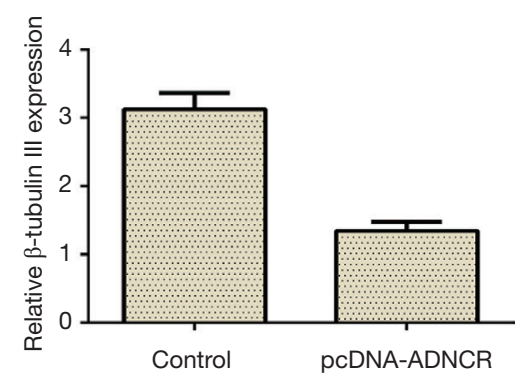

$E$

Control

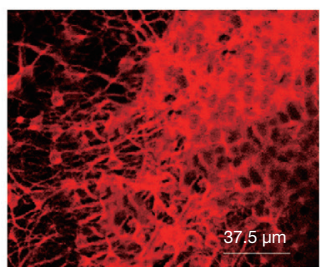

pcDNA-ADNCR

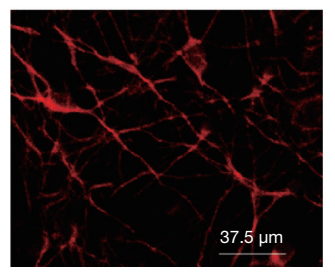

Figure 4 Ectopic expression of ADNCR promoted NSC proliferation and differentiation into neurons. (A) Increased expression of ADNCR promoted NSCs growth, as assessed by CCK-8 assay; (B) the expression of nestin was determined by qRT-PCR analysis; (C) $\beta$-Tubulin III expression was inhibited in neural induction cells treated with pcDNA-ADNCR; (D) the protein expression of $\beta$-Tubulin III was determined by western blot; (E) the immunocytochemical staining of $\beta$-Tubulin III also indicated that the overexpression of ADNCR suppressed NSC differentiation into neurons. *, $\mathrm{P}<0.05$; **, $\mathrm{P}<0.01$. ADNCR, adipocyte differentiation-associated long noncoding RNA; NSC, neural stem cell.

remains unknown. In this study, ADNCR expression was shown to decrease during the differentiation of NSCs into both neuron and astrocyte induction cells. The ectopic expression of ADNCR enhanced NSC proliferation and suppressed NSC differentiation into neurons.

Previous studies indicated that TCF3 played important functional roles associated with the modulation of neurogenesis (38). TCF3 acted as a suppressor of Wnt expression and functions as an activator of $\beta$-catenin (39). For instance, Kuwahara et al. (40) demonstrated that TCF3 expression was downregulated in cells that differentiate into neurons. TCF3 maintains populations by inhibiting the $\mathrm{Wnt} / \beta$-catenin signal pathway during neocortical development. Wang and colleagues found that miR-506-3p modulated NSC differentiation and proliferation by regulating TCF3 expression (3). In our study, we found that TCF3 expression decreased during the differentiation of NSCs into both neuron and astrocyte induction cells.
We used bioinformatics analysis to show that TCF3 is a potential target of miR-204-5p. In a dual-luciferase reporter assay, the ectopic expression of miR-204-5p decreased the luciferase activity of WT TCF3 3'-UTR but not Mut TCF3 3'-UTR. The overexpression of ADNCR inhibited the expression of miR-204-5p and enhanced the expression of TCF3. The ectopic expression of ADNCR enhanced NSC proliferation and suppressed NSC differentiation into neurons, partly via the regulation of TCF3 expression.

In summary, our data revealed that both ADNCR and TCF3 expression decreased during the differentiation of NSCs into both neuron and astrocyte induction cells, and the ectopic expression of ADNCR enhanced NSC proliferation and suppressed NSC differentiation into neurons, partly via the regulation of TCF3 expression. These data suggested that the use of ADNCR may represent a new strategy for expanding the interventions used to treat neurological disorders. 
A

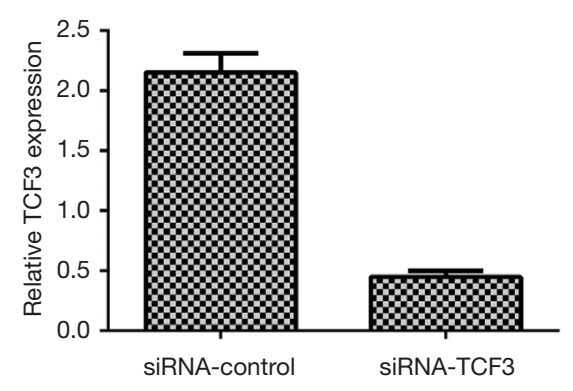

D

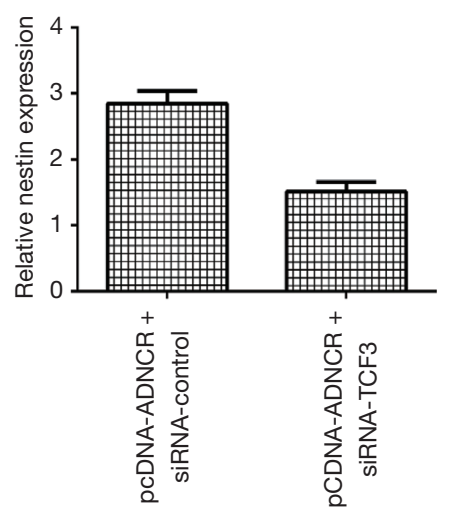

B

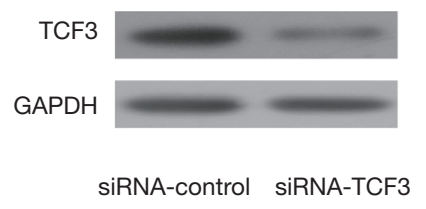

C

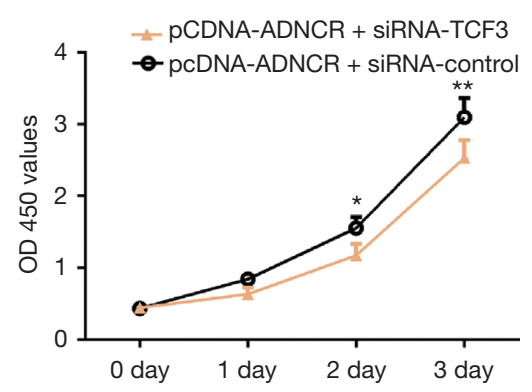

$\mathrm{F}$

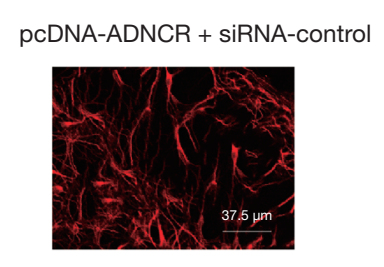

PCDNA-ADNCR + siRNA-TCF3

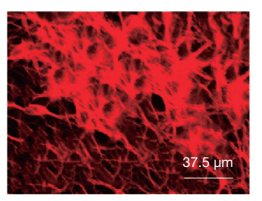

Figure 5 Inhibition of TCF3 expression abolishes the effects of ADNCR overexpression on NSC differentiation and proliferation. (A) The expression of TCF3 was analyzed by qRT-PCR assay; (B) the protein expression of TCF3 was determined by western blot; (C) CCK8 analysis results indicated that the knockdown TCF3 expression induced cell growth in ADNCR-overexpressing NSCs; (D) the expression of nestin was detected by qRT-PCR assay; (E) the expression of $\beta$-Tubulin III was determined by qRT-PCR assay; (F) immunocytochemical staining of $\beta$-Tubulin III also showed that the suppression of TCF3 enhanced NSC differentiation into neurons. *, $\mathrm{P}<0.05 ;{ }^{* *}, \mathrm{P}<0.01$. TCF3, transcription factor 3; ADNCR, adipocyte differentiation-associated long noncoding RNA.

\section{Acknowledgments}

Funding: This study was supported by the Natural Science Foundation of China (grant No. 81501117), the Natural Science Foundation of Guangdong Province (grant No. 2015A030310074, 2018A0303130307), Guangdong Basic and Applied Basic Research Foundation (gran No. 2019A1515011739) and R \& D Projects in Key Areas of Guangdong Province (grant No. 2018B030332001).

\section{Footnote}

Data Sharing Statement: Available at http://dx.doi. org/10.21037/atm-20-1068
Conflicts of Interest: All authors have completed the ICMJE uniform disclosure form (available at http://dx.doi. org/10.21037/atm-20-1068). The authors have no conflicts of interest to declare.

Ethical Statement: The authors are accountable for all aspects of the work in ensuring that questions related to the accuracy or integrity of any part of the work are appropriately investigated and resolved. The need for ethics approval and consent of the present study was waived by Third Affiliated Hospital of Sun Yat-sen University.

Open Access Statement: This is an Open Access article 
distributed in accordance with the Creative Commons Attribution-NonCommercial-NoDerivs 4.0 International License (CC BY-NC-ND 4.0), which permits the noncommercial replication and distribution of the article with the strict proviso that no changes or edits are made and the original work is properly cited (including links to both the formal publication through the relevant DOI and the license). See: https://creativecommons.org/licenses/by-nc-nd/4.0/.

\section{References}

1. Cui Y, Xiao Z, Han J, et al. MiR-125b orchestrates cell proliferation, differentiation and migration in neural stem/ progenitor cells by targeting Nestin. BMC Neurosci 2012;13:116.

2. Cardano M, Diaferia GR, Cattaneo M, et al. mSEL1L (Suppressor/enhancer Lin12-like) protein levels influence murine neural stem cell self-renewal and lineage commitment. J Biol Chem 2011;286:18708-19.

3. Wang Y, Jiaqi C, Zhaoying C, et al. MicroRNA-506-3p regulates neural stem cell proliferation and differentiation through targeting TCF3. Gene 2016;593:193-200.

4. Liu C, Teng ZQ, McQuate AL, et al. An epigenetic feedback regulatory loop involving microRNA-195 and MBD1 governs neural stem cell differentiation. PLoS One 2013;8:e51436.

5. Li X, Feng R, Huang C, et al. MicroRNA-351 regulates TMEM 59 (DCF1) expression and mediates neural stem cell morphogenesis. RNA Biol 2012;9:292-301.

6. Guo F, Han X, Zhang J, et al. Repetitive transcranial magnetic stimulation promotes neural stem cell proliferation via the regulation of MiR-25 in a rat model of focal cerebral ischemia. PLoS One 2014;9:e109267.

7. Feliciano DM, Zhang S, Nasrallah CM, et al. Embryonic cerebrospinal fluid nanovesicles carry evolutionarily conserved molecules and promote neural stem cell amplification. PLoS One 2014;9:e88810.

8. Brett JO, Renault VM, Rafalski VA, et al. The microRNA cluster miR-106b 25 regulates adult neural stem/ progenitor cell proliferation and neuronal differentiation. Aging (Albany NY) 2011;3:108-24.

9. Aranha MM, Santos DM, Sola S, et al. miR-34a regulates mouse neural stem cell differentiation. PLoS One 2011;6:e21396.

10. Garg N, Po A, Miele E, et al. microRNA-17-92 cluster is a direct Nanog target and controls neural stem cell through Trp53inp1. EMBO J 2013;32:2819-32.

11. González-Gómez P, Sanchez P, Mira H. MicroRNAs as regulators of neural stem cell-related pathways in glioblastoma multiforme. Mol Neurobiol 2011;44:235-49.

12. Goustard-Langelier B, Koch M, Lavialle M, et al. Rat neural stem cell proliferation and differentiation are durably altered by the in utero polyunsaturated fatty acid supply. J Nutr Biochem 2013;24:380-7.

13. Morgado AL, Xavier JM, Dionisio PA, et al. MicroRNA34a Modulates Neural Stem Cell Differentiation by Regulating Expression of Synaptic and Autophagic Proteins. Mol Neurobiol 2015;51:1168-83.

14. Zhuang Y, Jiang HY, Li H, et al. Down-regulation of long non-coding RNA AFAP1-AS1 inhibits tumor cell growth and invasion in lung adenocarcinoma. Am J Transl Res 2017;9:2997-3005.

15. Zhou DD, Liu XF, Lu CW, et al. Long non-coding RNA PVT1: Emerging biomarker in digestive system cancer. Cell Prolif 2017;50:e12398.

16. Yu N, Liang Y, Zhu H, et al. CsA Promotes XIST Expression to Regulate Human Trophoblast Cells Proliferation and Invasion Through miR-144/Titin Axis. J Cell Biochem 2017;118:2208-18.

17. Wang Z, Yuan JW, Li L, et al. Long non-coding RNA XIST exerts oncogenic functions in human glioma by targeting miR-137. Am J Transl Res 2017;9:1845-55.

18. Wang $X$, Lu X, Geng Z, et al. LncRNA PTCSC3/miR574-5p Governs Cell Proliferation and Migration of Papillary Thyroid Carcinoma via Wnt/-Catenin Signaling. J Cell Biochem 2017;118:4745-52.

19. Wang X, Lv G, Li J, et al. LncRNA-RP11-296A18.3/miR138/HIF1A Pathway Regulates the Proliferation ECM Synthesis of Human Nucleus Pulposus Cells (HNPCs). J Cell Biochem 2017;118:4862-71.

20. Ma X, Qi S, Duan Z, et al. Long non-coding RNA LOC554202 modulates chordoma cell proliferation and invasion by recruiting EZH2 and regulating miR-31 expression. Cell Prolif 2017;50:e12388.

21. Liao Y, Shen L, Zhao H, et al. LncRNA CASC2 Interacts With miR-181a to Modulate Glioma Growth and Resistance to TMZ Through PTEN Pathway. J Cell Biochem 2017;118:1889-99.

22. Zhang S, Dong X, Ji T, et al. Long non-coding RNA UCA1 promotes cell progression by acting as a competing endogenous RNA of ATF2 in prostate cancer. Am J Transl Res 2017;9:366-75.

23. Xi Y, Jiang T, Wang W, et al. Long non-coding HCG18 promotes intervertebral disc degeneration by sponging miR-146a-5p and regulating TRAF6 expression. Sci Rep 2017;7:13234. 
24. Qiao Y, Peng CL, Li J, et al. LncRNA MALAT1 is Neuroprotective in a Rat Model of Spinal Cord Ischemiareperfusion Injury Through miR-204 Regulation. Curr Neurovasc Res 2018;15:211-9.

25. Xie N, Qi J, Li S, et al. Upregulated lncRNA small nucleolar RNA host gene 1 promotes 1-methyl-4phenylpyridinium ion-induced cytotoxicity and reactive oxygen species production through miR-15b-5p/GSK3 $\beta$ axis in human dopaminergic SH-SY5Y cells. J Cell Biochem 2019;120:5790-801.

26. Zheng J, Yi D, Liu Y, et al. Long nonding RNA UCA1 regulates neural stem cell differentiation by controlling miR-1/Hes1 expression. Am J Transl Res 2017;9:3696-704.

27. Yen YP, Hsieh WF, Tsai YY, et al. Dlk1-Dio3 locusderived lncRNAs perpetuate postmitotic motor neuron cell fate and subtype identity. Elife 2018;7:e38080.

28. Knauss JL, Miao N, Kim SN, et al. Long noncoding RNA Sox2ot and transcription factor YY1 co-regulate the differentiation of cortical neural progenitors by repressing Sox2. Cell Death Dis 2018;9:799.

29. Li M, Sun X, Cai H, et al. Long non-coding RNA ADNCR suppresses adipogenic differentiation by targeting miR-204. Biochim Biophys Acta 2016;1859:871-82.

30. Shi X, Yan C, Liu B, et al. miR-381 Regulates Neural Stem Cell Proliferation and Differentiation via Regulating Hes1 Expression. PLoS One 2015;10:e0138973.

31. Suksuphew S, Noisa P. Neural stem cells could serve as a therapeutic material for age-related neurodegenerative diseases. World J Stem Cells 2015;7:502-11.

32. Rolando C, Taylor V. Neural stem cell of the hippocampus: development, physiology regulation, and dysfunction in disease. Curr Top Dev Biol 2014;107:183-206.

Cite this article as: Long L, Zeng C, Chen H, Zhou T, Wu L, Cai X. ADNCR modulates neural stem cell differentiation and proliferation through the regulation of TCF3 expression. Ann Transl Med 2020;8(15):927. doi: 10.21037/atm-20-1068
33. Pham JT, Gallicano GI. Specification of neural cell fate and regulation of neural stem cell proliferation by microRNAs. Am J Stem Cells 2012;1:182-95.

34. Li Z, Li XY, Chan MTV, et al. Melatonin antagonizes interleukin-18-mediated inhibition on neural stem cell proliferation and differentiation. J Cell Mol Med 2017;21:2163-71.

35. Zhang L, Xue Z, Yan J, et al. LncRNA Riken-201 and Riken-203 modulates neural development by regulating the Sox6 through sequestering miRNAs. Cell Prolif 2019;52:e12573.

36. Li Y, Guo B, Yang R, et al. A novel long noncoding RNA lnc158 promotes the differentiation of mouse neural precursor cells into oligodendrocytes by targeting nuclear factor-IB. Neuroreport 2018;29:1121-8.

37. Winzi M, Vila NC, Paszkowski-Rogacz M, et al. The long noncoding RNA lncR492 inhibits neural differentiation of murine embryonic stem cells. PLoS One 2018;13:e0191682.

38. Kim S, Chung A, Kim D, et al. Tcf3 function is required for the inhibition of oligodendroglial fate specification in the spinal cord of zebrafish embryos. Mol Cells 2011;32:383-8.

39. Liu W, Liu Y, Guo T, et al. TCF3, a novel positive regulator of osteogenesis, plays a crucial role in miR-17 modulating the diverse effect of canonical Wnt signaling in different microenvironments. Cell Death Dis 2013;4:e539.

40. Kuwahara A, Sakai H, Xu Y, et al. Tcf3 represses Wnt$\beta$-catenin signaling and maintains neural stem cell population during neocortical development. PLoS One 2014;9:e94408. 